\title{
Landau-like states in the magneto-optical spectrum of a shallow donor impurity: Theory versus experiment
}

\author{
P. W. Barmby, J. L. Dunn, and C. A. Bates \\ Department of Physics, University of Nottingham, University Park, Nottingham, NG7 2RD, United Kingdom \\ T. O. Klaassen \\ Faculty of Applied Physics, Delft University of Technology, P.O. Box 5046, 2600 GA Delft, The Netherlands
}

(Received 6 March 1996)

\begin{abstract}
A calculation of the energy levels and the wave functions for a shallow donor impurity in an applied magnetic field is put forward. The behavior of the donor electron in directions perpendicular to the field is described by a basis set of Landau wave functions, and a variational procedure is used to generate approximate forms for the wave function along the field axis. Theoretical values for the ionization energies of the impurity are calculated and a comparison with both existing and new experimental data, also reported here, is made for impurities in bulk GaAs. The model is used to obtain hydrogenlike states of the impurity as well as the so-called "metastable" or " autoionizing"' Landau-like states. A comparison with a "hydrogenic" method of calculation is used to highlight the validity of the model. Also, transition probabilities from the ground to excited donor states are deduced. The results obtained are used to identify the various peaks observed in experimental spectra. Differences between theory and experiment are attributed to the occurrence of Fano resonances. A discussion of the behavior of the impurity in an applied magnetic field calls into question the expected ordering of the energy levels of such a system. [S0163-1829(96)05735-9]
\end{abstract}

\section{INTRODUCTION}

Photospectroscopy experiments, carried out on $n$-type GaAs in applied magnetic fields, have revealed transitions involving not only the much-studied hydrogenlike states of donor impurities, but also transitions involving the so-called metastable, or autoionizing impurity states. ${ }^{1-3}$ These latter states are associated with the Landau levels of a free electron, modified by the Coulomb interaction between the donor ion and the electron, and as such are observed only in finite magnetic fields. Henceforth, such states will be referred to as Landau-like states.

Although there has been extensive work carried out on the theoretical modeling of the hydrogenlike states of donor impurities in GaAs, there has been little work carried out on the modeling of the Landau-like states. Simola and Virtamo ${ }^{4}$ put forward a detailed theoretical method for modeling the Landau-like states of a hydrogen atom in a strong magnetic field which used a numerical procedure to ascertain the required energies. Friedrich and co-workers also used numerical methods to obtain approximate solutions for the Schrödinger equation. ${ }^{5-7}$ The main drawback to these methods is that the numerical nature of the calculations means that no analytical wave functions can be obtained. Thus transition probabilities cannot be computed. Also, transitions involving the Landau-like states have been observed at energies fairly close to that of longitudinal-optical phonons, and anticrossing effects involving the electron-phonon interaction with these donor states have been clearly seen. It is highly desirable for any model of this electron-phonon interaction to describe the Landau-like states in an analytic form.

In earlier work, Zhilich and Monozon ${ }^{8}$ used a variational procedure to calculate the energies of Landau-like states of shallow donors which did yield an approximate form for the wave functions of these states. However, their method applies only for extreme values of applied magnetic field and is complicated by the use of Whittaker functions as trial basis states. Golubev et al. ${ }^{9}$ also used a variational method to study Landau-like states in GaAs, but again they used a complicated trial wave function involving a summation over many basis states. In the extensive work on the hydrogen atom in strong magnetic fields, Friedrich ${ }^{10}$ used initially a wave function comprising a summation over many Gaussians to describe the behavior of the electron along the field axis, together with a summation over a series of Landau states to model the mixing of these states due to the presence of the Coulomb interaction. The autoionization states of the hydrogen atom in intense magnetic fields were also analyzed by Bhattacharya and $\mathrm{Chu}^{11}$ and by Greene. ${ }^{12}$ A review of this work by Friedrich and Wintgen ${ }^{13}$ appeared in 1989 with the emphasis on chaotic motion. None of these methods results in wave functions in a readily usable form. Also, very little work has been undertaken on the excited states.

The main objective of this paper is to develop a theoretical model that uses a variational approach to obtain simple expressions for the wave functions of the Landau-like states. Results are also obtained for the hydrogenlike states, and their validity is discussed. The method is based on the original work of Wallis and Bowlden ${ }^{14}$ but it is expanded considerably to describe a wider range of states. Results are obtained for many highly excited Landau-like states for the first time. A secondary objective of the paper is to report new experimental studies of the far-infrared spectra obtained from samples of GaAs doped with silicon that give direct support to the new model. Many of the features which were previously unidentified but which were observed in these and other recent experimental studies of shallow donor states in $\mathrm{GaAs}^{15}$ can be identified as transitions involving Landau-like 
states with quantum numbers $N \geqslant 10$.

Section II gives details of the new model, and Sec. III describes new infrared experiments carried out on silicondoped GaAs samples. The transition energies to Landau-like states are measured and, from these measurements, the ionization energies are deduced for a range of magnetic fields. This necessitates correcting the raw data to take into account the effects of nonparabolicity in the conduction band, the electron-phonon interaction, and the central cell correction. Sections IV and V include detailed discussions of the results and their implications. Section VI discusses the ordering of the Landau-like states, and Sec. VII concludes with a brief mention of other cases of Landau-like behavior of impurities in multi-quantum-well systems.

\section{MODEL}

The Hamiltonian for the electron of effective mass $m^{*}$ associated with a hydrogenic impurity in an applied magnetic field $B$ can be written in the form

$$
\mathcal{H}=\mathcal{H}_{\rho \phi}+\mathcal{H}_{z}+\mathcal{H}_{C}
$$

where

$$
\begin{gathered}
\mathcal{H}_{\rho \phi}=-\frac{1}{\rho} \frac{\partial}{\partial \rho}\left(\rho \frac{\partial}{\partial \rho}\right)-\frac{1}{\rho^{2}} \frac{\partial^{2}}{\partial \phi^{2}}+\gamma l_{z}+\frac{\gamma^{2}}{4} \rho^{2}, \\
\mathcal{H}_{z}=-\frac{\partial^{2}}{\partial z^{2}},
\end{gathered}
$$

and $\mathcal{H}_{C}=-2 / \sqrt{\rho^{2}+z^{2}}$.

As the physical system has cylindrical symmetry about the applied-magnetic-field direction (defined to be the $z$ axis), cylindrical coordinates and a symmetric form for the gauge of the field have been used. All lengths have been expressed in units of effective Bohr radii $a_{0}^{*}\left(=\hbar^{2} 4 \pi \epsilon / m^{*} e^{2}\right)$ and all energies in effective Rydbergs $\mathcal{R}^{*}\left(=e^{2} / 8 \pi \epsilon a_{0}^{*}\right.$, where $\epsilon$ is the dielectric constant of the semiconductor material). The quantity $\gamma$ is a dimensionless form for the magnitude of the magnetic field where

$$
\gamma=\frac{(4 \pi \epsilon)^{2} \hbar^{3}}{m^{* 2} e^{3}} B,
$$

and $l_{z}$ is the operator associated with the $z$ component of the orbital angular momentum of the donor electron. In writing down the Hamiltonian, we have assumed that the effective mass $m^{*}$ can be treated as a constant for the range of magnetic fields and electron energies to be considered.

Initially, a convenient form for the wave function $\Psi$ of the donor electron in the magnetic field is ascertained by utilizing the adiabatic approach. It is assumed that the effect of the magnetic field is far greater than that of the Coulomb attraction between the electron and the impurity nucleus in the direction perpendicular to the field so that the Coulomb term does not mix states with different values of $N$. Thus the behavior of the electron in the $\rho$ and $\phi$ directions can be treated separately from that in the $z$ direction. Therefore we write

$$
\Psi_{N m \nu}=\Phi_{N m}(\rho, \phi) \times f_{\nu}(z),
$$

where $\Phi_{N m}(\rho, \phi)$ and $f_{\nu}(z)$ are the components of the wave function perpendicular and parallel to the magnetic field, respectively. The perpendicular component of the wave function is given by the Landau wave function

$$
\begin{aligned}
\Phi_{N m}(\rho, \phi)= & \sqrt{\frac{\gamma}{2 \pi}}\left[\frac{n_{\rho} !}{\left(n_{\rho}+|m|\right) !}\right]^{1 / 2} \\
& \times e^{-(1 / 2) \xi} \xi^{(1 / 2)|m|} L_{n_{\rho}}^{|m|}(\xi) e^{i m \phi},
\end{aligned}
$$

where $m$ is the quantum number associated with the $z$ component of the orbital angular momentum of the electron, $n_{\rho}$ is a non-negative integer, $\xi=\frac{1}{2} \gamma \rho^{2}$, and $L_{n_{\rho}}^{|m|}(\xi)$ is a Laguerre polynomial. The energy of the corresponding Landau level is given by

$$
E_{N}=2 \gamma\left(N+\frac{1}{2}\right)
$$

where $N=n_{\rho}+\frac{1}{2}(|m|+m)$ is also a non-negative integer. Each Landau state is thus defined completely by the quantum numbers $N$ and $m$. The subscript $\nu$ is an additional quantum number denoting the number of nodes of the wave function along the magnetic field axis $(\nu=0,2,4 \ldots$ for even parity states, $\nu=1,3,5 \ldots$ for odd parity states). In this study, we restrict our calculations to the $\nu=0,1$, and 2 states as these are the ones most likely to be observed in experiments.

An approximate analytic form for the perpendicular component of the wave function $f_{\nu}(z)$ is deduced using a variational procedure. The form of $f_{\nu}(z)$ chosen is the same as that used by Wallis and Bowlden, ${ }^{14}$ namely, an orthogonal set of Hermite polynomials. In particular, the form of $f_{\nu}(z)$ for $\nu=0,1$, and 2 is given by

$$
\begin{gathered}
f_{0}(z)=\left(\frac{2 \alpha_{0}}{\pi}\right)^{1 / 4} e^{-\alpha_{0} z^{2}} \\
f_{1}(z)=\left(\frac{32 \alpha_{1}^{3}}{\pi}\right)^{1 / 4} z e^{-\alpha_{1} z^{2}} \\
f_{2}(z)=\left(\frac{2 \alpha_{2}}{\pi}\right)^{1 / 4} \frac{2 \alpha_{2}}{\left(3 \alpha_{0}^{2}+2 \alpha_{0} \alpha_{2}+3 \alpha_{2}^{2}\right)^{1 / 2}} \\
\quad \times\left[2\left(\alpha_{0}+\alpha_{2}\right) z^{2}-1\right] e^{-\alpha_{2} z^{2}} .
\end{gathered}
$$

The $\alpha_{\nu}$ are the variational parameters, and the prefactors ensure that the functions are normalized over the $z$ direction.

To find the energy of an impurity state of given $N, m$, and $\nu$, the parameters $\alpha_{\nu}$ are varied in order to minimize the energy $E_{N m \nu}$ of the states $\Psi_{N m \nu}$ where

$$
\begin{aligned}
E_{N m \nu}= & \left\langle\Psi_{N m \nu}|\mathcal{H}| \Psi_{N m \nu}\right\rangle=2 \gamma\left\langle N+\frac{1}{2}\right\rangle+F_{\nu} \\
& -4 \pi \int_{-\infty}^{\infty} \int_{0}^{\infty} \frac{\left|\Phi_{N, m}(\rho, \phi)\right|^{2}\left|f_{\nu}(z)\right|^{2}}{\left(\rho^{2}+z^{2}\right)^{1 / 2}} \rho d \rho d z
\end{aligned}
$$

and where 


$$
\begin{gathered}
F_{0}=\alpha_{0}, \\
F_{1}=3 \alpha_{1}, \\
F_{2}=\frac{\alpha_{2}}{\left(3 \alpha_{0}^{2}+2 \alpha_{0} \alpha_{2}+3 \alpha_{2}^{2}\right)}\left(7 \alpha_{0}^{2}+18 \alpha_{0} \alpha_{2}+15 \alpha_{2}^{2}\right) .
\end{gathered}
$$

This variational procedure also generates the wave function $\Psi_{N m \nu}$. The integral in Eq. (8) can be calculated by computational means.

The adiabatic approximation used above is reasonable at high magnetic fields as the Coulomb interaction is a small perturbation on the behavior of the donor electron in the applied field. However, when the donor electron is tightly bound to the impurity ion (as in the case of the hydrogenlike states of the impurity), the approximation will be less applicable. Greater accuracy may be obtained by allowing states of differing $N$ to admix via the Coulomb interaction. The states $\Psi_{N m \nu}$ with different $N$ but given $m$ and $\nu$ can be used as basis states for a new state $\Psi_{N^{\prime} m \nu}$ describing a range of values of $N$. The subscript $N^{\prime}$ denotes the Landau level with which the impurity state is predominantly associated.

Allowing for the mixing of states of differing $N$, a typical matrix expression for an element of the Hamiltonian is given by

$$
\left\langle\Psi_{N_{2} m \nu}|\mathcal{H}| \Psi_{N_{1} m \nu}\right\rangle=\delta_{N_{1} N_{2}}\left[2 \gamma\left[N+\frac{1}{2}\right]+F_{\nu}\right]-2 \int_{0}^{2 \pi} \int_{-\infty}^{\infty} \int_{0}^{\infty} \frac{\Phi_{N_{1}, m}(\rho, \phi) \Phi_{N_{2},}^{*}(\rho, \phi) f_{\nu}^{N_{1} m}(z) f_{\nu}^{N_{2} m}(z)}{\left(\rho^{2}+z^{2}\right)^{1 / 2}} \rho d \rho d z d \phi,
$$

where $\delta_{N_{1} N_{2}}$ is the Kronecker delta function and $f_{\nu}^{N m}(z)$ is the $z$ component of the basis state $\Psi_{N m \nu}$ calculated previously for a given $N$ and $m$. By calculating the matrix of the Hamiltonian $\mathcal{H}$ and diagonalizing it to obtain the energies $E_{N^{\prime} m \nu}$, a more accurate estimation of the energies of the impurity states and its wave functions can be obtained. It must be noted, however, that since the basis states used in the linear combination for the state $\Psi_{N^{\prime} m \nu}$ are not true eigenstates of the Schrödinger equation but simply approximate states, this improvement tends to lead to a lowering of the energy of states associated with the lower values of $N^{\prime}$ only. This latter method is therefore used only to improve the modeling of the hydrogenic states of the impurity.

\section{EXPERIMENTAL DETAILS}

Two different samples were used in the far-infrared experiments. Both samples are approximately $10-\mu \mathrm{m}$-thick epitaxial layers of $n$-GaAs on a semi-insulating substrate, intentionally doped with $\mathrm{Si}$ and grown by molecular-beam epitaxy (MBE) at the Philips Research Laboratory, Redhill, UK. For the first sample, the donor and acceptor concentrations were determined (at source and by temperaturedependent Hall measurements) to be $5 \times 10^{14}$ and $1 \times 10^{14}$ $\mathrm{cm}^{-3}$, respectively. No direct determination of the impurity concentrations in the second sample was available, but comparisons of the spectra and the $T=77 \mathrm{~K}$ mobility data from both samples suggests that the donor concentrations are very similar. From an inspection of the far-infrared line shape of the $1 s_{0}-2 p_{ \pm 1}$ transitions, the concentration of donors other than $\mathrm{Si}$ in both samples is estimated to be no greater than $5 \%$.

In the experiments, a conventional optically pumped farinfrared molecular gas laser was used. The sample was placed in the centre of a superconducting magnet and the spectra were recorded by measuring the far-infrared induced conductivity of the sample while sweeping the magnetic field. The majority of the experimental data presented here originate from previous work on the first sample ${ }^{2,15,16}$ but additional data have been obtained from recent experiments on the second sample.

In order to present the theoretical data in as general a way as possible (i.e., applicable for the hydrogen atom as well as for shallow donors in various semiconducting materials), dimensionless field and energy units have been used. Thus, in order to present the experimental data, similar dimensionless units must be used for comparison. The latter have also to be stripped of its material-specific character. Thus the data obtained experimentally from the silicon donor in GaAs have been corrected for the small nonparabolicity (NPB) of the conduction band and the influence of electron-phonon (EP) interaction. Both corrections are needed for GaAs; each leads to a magnetic field and/or energy-dependent effective mass of the conduction electrons. The correction for nonparabolicity has been obtained from the expression obtained by Vrehen ${ }^{17}$ namely, that the corrected energy $E$ is given by the expression $E=E_{0}\left(1-\delta E_{0} / E_{g}\right)$, where $E_{0}$ is the energy calculated for a parabolic band, $E_{g}$ is the band-gap energy, and the parameter $\delta=0.83$. The influence of the nonresonant electron-phonon interaction has been accounted for using the low-field analytical expressions given by Peeters and Devreese $^{18}$ with the electron-phonon interaction constant $\alpha=0.066$.

The far-infrared experiments measure the energy difference between the ground state $\psi_{000}$ and an excited donor state $\Psi_{N^{\prime} m \nu}$. In order to derive the ionization energy of the donor states (i.e., the energy difference between the Landau level $N$ and the state $\Psi_{N^{\prime} m \nu}$ ), the small donor-specific, central cell correction (CCC) for the ground-state energy must also to be taken into account. This field-dependent energy correction may be written in the form $\Delta E=(0.7$ $\left.+6 \times 10^{-3} B^{2}\right) \mathrm{cm}^{-1}$; this expression has been derived from an analysis of data on the field dependence of transitions to the $2 p_{-1}, 2 p_{0}$, and $3 p_{-1}$ states in GaAs. ${ }^{19}$ Using all the above corrections, the true ionization energies $E_{\text {ion }}$ of the 


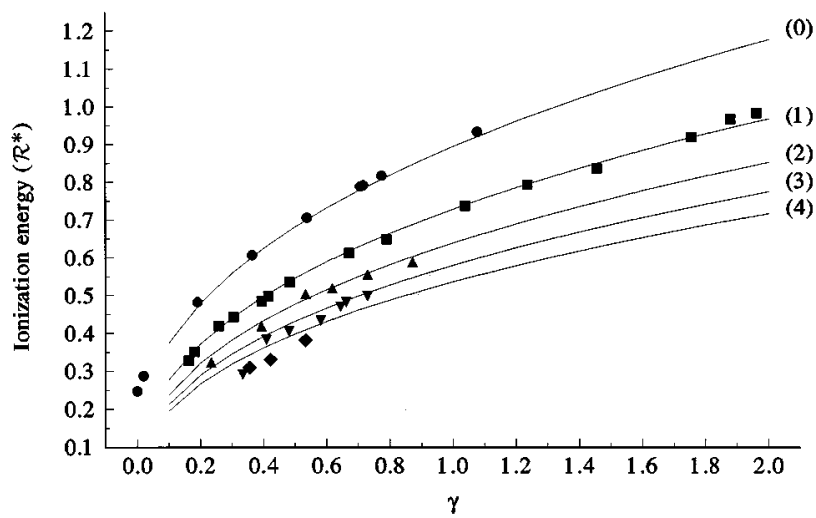

FIG. 1. Theoretical values for the ionization energies of shallow donor states for a range of applied magnetic fields (solid lines) and the corresponding ionization energies calculated from experimental data for the $(N,-1,0)$ states.

donor states have been obtained from the experimental transition energies $E_{\text {exp }}$ using

$$
E_{\mathrm{ion}}=\left(N+\frac{1}{2}\right) \hbar \omega_{\mathrm{CR}}-E_{000}-E_{\mathrm{exp}}+\Delta E_{\mathrm{NPB}}+\Delta E_{\mathrm{EP}}+\Delta E_{\mathrm{CCC}}
$$

in an obvious notation. Here $\hbar \omega_{\mathrm{CR}}\left(=13.98 \mathrm{~cm}^{-1}\right)$ is the experimentally determined value for the cyclotron resonance frequency in the low-field limit. From this value, a zero-field effective mass of $m^{*}\left(=0.0668 m_{e}\right)$ is obtained. Finally, the field and energy values have been transformed to dimensionless units using the zero-field values $\mathcal{R}^{*}=46.11 \mathrm{~cm}^{-1}$ and $\gamma=B / B_{0}$ where $B_{0}=6.59 \mathrm{~T}$. All finite field data have been corrected for the increase of $m^{*}$ with field and/or energy causing an increase in both $\mathcal{R}^{*}$ and $B_{0}$.

\section{THE IONIZATION ENERGIES}

The ionization energies derived from both the model and the experiments (corrected as outlined above) are plotted together in Figs. 1-4 for a range of $\gamma$ values. The theoretical energies of the impurity states of given $N^{\prime}, m$, and $\nu$ were calculated as detailed above, and the corresponding values for the ionization energies were deduced from the expression $2 \gamma\left(N^{\prime}+\frac{1}{2}\right)-E_{N^{\prime} m \nu}$. In each case, the value of $N^{\prime}$ of the state of given $m$ and $\nu$ is given in brackets at the sides of the figures. From the figures, it can be seen that the model predicts ionization energies that closely match those obtained

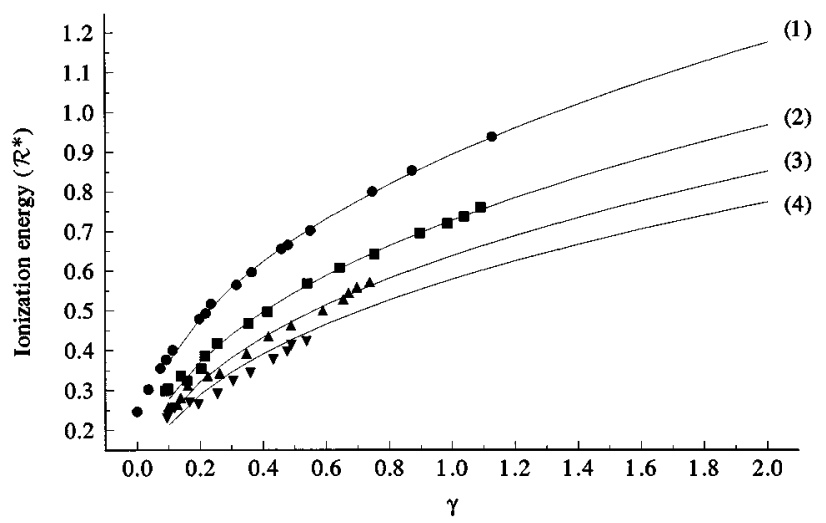

FIG. 2. As Fig. 1, but for the $(N, 1,0)$ states.

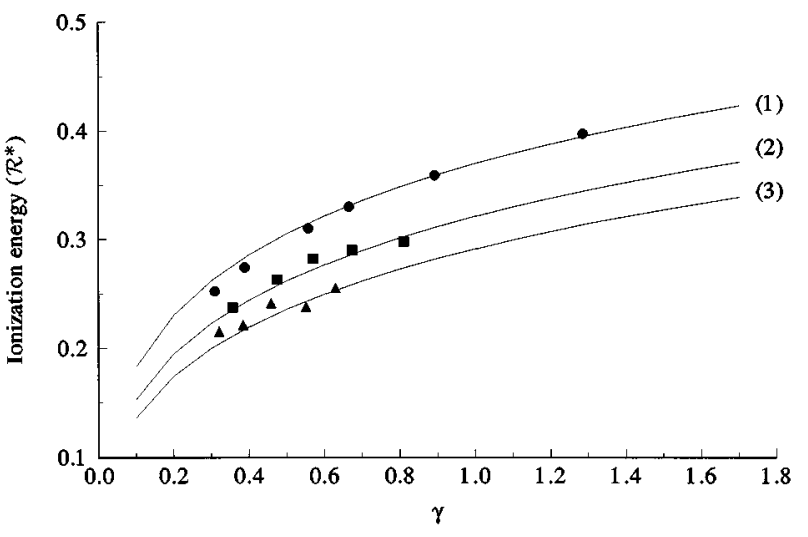

FIG. 3. As Fig. 1, but for the $(N, 0,1)$ states.

experimentally. The agreement between theory and experiment is best at the higher values of the magnetic field $\gamma$, where we would expect a method based on the adiabatic approximation to be more accurate. However, the agreement for lower magnetic fields is still reasonable. The larger deviations between theory and experiments which appears in Fig. 4 for the $(N, 1,2)$ states is attributed mainly to the difficulty in obtaining accurate values for the experimental data.

The agreement between experiment and theory is best for the lower values of $N^{\prime}$, with an increasing discrepancy for increasing values of $N^{\prime}$. However, we would have expected the fit to be poorer at lower values of $N^{\prime}$ where the electron is more strongly bound to the impurity ion. We will consider this point again at the end of Sec. V.

The theoretical model was developed for the excited Landau-like states, where the adiabatic approximation is expected to be most applicable. However, results can also be produced for hydrogenlike states. To examine the range of validity of such results, the calculated ionization energies have been compared with the numerical values obtained by Makado and $\mathrm{McGill}^{20}$ using a hydrogenlike model. The two sets of ionization energies are given in Table I for a range of different states and $\gamma$ values. It can be seen that for larger magnetic fields and for the higher-energy excited states, where the Coulomb interaction is not so dominant, the differences between the two sets of results is as little as $1 \%$ or less. Our Landau-like approach, therefore, is seen to compare quite favorably with the hydrogenic method. However, the model gives quite different values at lower values of the

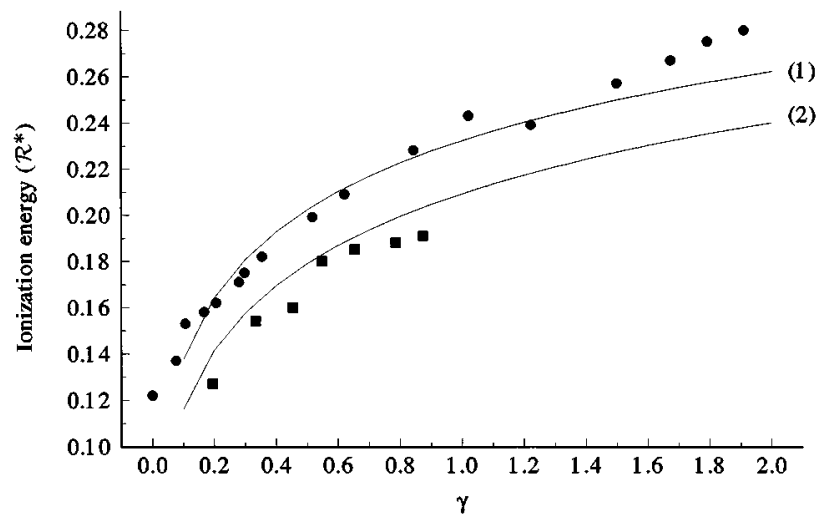

FIG. 4. As Fig. 1, but for the $(N, 1,2)$ states. 
TABLE I. Comparison of the ionization energies of hydrogenlike states obtained using the present theoretical method and those calculated from the hydrogenic method of Makado and McGill (given in parentheses).

\begin{tabular}{|c|c|c|c|c|c|c|c|}
\hline$N$ & $m$ & $\nu$ & $\gamma$ & 0.2 & 0.5 & 1.0 & 2.0 \\
\hline 0 & 0 & 0 & & $0.9066(1.18041)$ & $1.1977(1.39403)$ & $1.6619(1.50000)$ & $2.0438(1.89820)$ \\
\hline 0 & 0 & 1 & & $0.3390(0.37024)$ & $0.4217(0.44929)$ & $0.4901(0.51954)$ & $0.5597(0.59432)$ \\
\hline 0 & 0 & 2 & & $0.2058(0.29788)$ & $0.2451(0.30154)$ & $0.2749(0.32088)$ & $0.3033(0.34783)$ \\
\hline 1 & 1 & 0 & & $0.4797(0.50106)$ & $0.6804(0.69892)$ & $0.8938(0.91311)$ & $1.1767(1.19896)$ \\
\hline 1 & 1 & 1 & & $0.2545(0.26357)$ & $0.3321(0.34268)$ & $0.3994(0.41313)$ & $0.4715(0.49047)$ \\
\hline 1 & 1 & 2 & & $0.1641(0.18168)$ & $0.2023(0.21872)$ & $0.2323(0.25095)$ & $0.2622(0.28488)$ \\
\hline 2 & 2 & 0 & & $0.3575(0.36262)$ & $0.5231(0.52878)$ & $0.6991(0.70609)$ & $0.9329(0.94232)$ \\
\hline 2 & 2 & 1 & & $0.2147(0.21900)$ & $0.2870(0.29303)$ & $0.3513(0.35993)$ & $0.4220(0.43488)$ \\
\hline 2 & 2 & 2 & & $0.1441(0.15166)$ & $0.1811(0.19053)$ & $0.2108(0.22309)$ & $0.2409(0.25734)$ \\
\hline 3 & 3 & 0 & & $0.2981(0.30017)$ & $0.4425(0.44522)$ & $0.5961(0.59986)$ & $0.8009(0.80559)$ \\
\hline
\end{tabular}

magnetic field and for states having lower values for $N^{\prime}, m$, and $\nu$. It is apparent that the adiabatic approximation used in our model is no longer applicable for these strongly bound impurity states, where the Coulomb interaction is obviously more dominant. Alternative approaches, such as that of Dunn and Pearl, ${ }^{21}$ should be used for these states.

\section{FURTHER ANALYSES OF THE SPECTRA}

One of the advantages of the model presented here is that it uses a simple variational procedure to determine a suitable form for the wave function of the Landau-like states along the $z$ axis. This means that analytical forms for the wave functions can be obtained and so used in further calculations. For example, this model has been used to examine the effects of electron-LO-phonon coupling on the energies of Landaulike impurity states in bulk GaAs. ${ }^{16}$ The wave functions can also be used to calculate the transition probabilities from the ground state of the impurity to the excited Landau-like states to assist in the identification of the transitions observed in the experimental spectra. This will be considered now.

\section{A. Transition probabilities}

For the case in which the laser radiation is applied along the axis of the sample (Faraday geometry), the transition probability from the ground state $\Psi_{g}=\Psi_{000}$ of the impurity to an excited state $\Psi_{N^{\prime} m \nu}$ is proportional to $\left|\left\langle\Psi_{N^{\prime} m \nu}|x| \Psi_{g}\right\rangle\right|^{2}$. This is nonzero only when the quantum number $m$ associated with the excited state is \pm 1 and $\Psi_{N^{\prime} m \nu}$ is an even parity state. When the radiation is applied in a direction perpendicular to the field (Voigt geometry) the transition probability is proportional to either $\left|\left\langle\Psi_{N^{\prime} m \nu}|x| \Psi_{g}\right\rangle\right|^{2}$ or $\left|\left\langle\Psi_{N^{\prime} m \nu}|z| \Psi_{g}\right\rangle\right|^{2}$. These matrix elements are nonzero either (i) when $m= \pm 1$ and $\Psi_{N^{\prime} m \nu}$ is an even parity state or (ii) when $m=0$ and $\Psi_{N^{\prime} m \nu}$ is an odd parity state.

The predicted peaks in the spectra of allowed transitions at a fixed wavelength have been obtained as a function of magnetic field $B$. An example of such a theoretical spectrum is given in Fig. 5; it corresponds to a laser wavelength of 70 $\mu \mathrm{m}$ (equivalent to an energy of $142.86 \mathrm{~cm}^{-1}$ ). Each transition is plotted as a line at the value of magnetic field which corresponds to this transition energy, and the height of the line is proportional to the theoretical transition probability. The ground state $\psi_{000}$ and the low-lying hydrogenlike states $\psi_{110}$ and $\psi_{112}$ were modeled using the hydrogenic method of Dunn and Pearl ${ }^{21}$ as discussed above. Also, in order to simplify the calculations, results were obtained using the adiabatic limit where the states have a particular value for $N$, rather than a linear combination of values. For the purposes of the plot, the calculation was again carried out for a shallow donor impurity in bulk GaAs and the magnetic field and the transition energies in the calculation were expressed in terms of standard units.

Figure 5 also shows two examples of experimental spectra at a laser wavelength of $70 \mu \mathrm{m}$ for comparison with the theory; one example is a spectrum obtained in the Faraday configuration and the other was obtained in the Voigt configuration. On comparing the experimental and theoretical spectra, the various transitions in the experimental spectra can be identified. To aid the identification, line 1, which arises from a transition to the $(1,1,0)$ state, has been scaled to

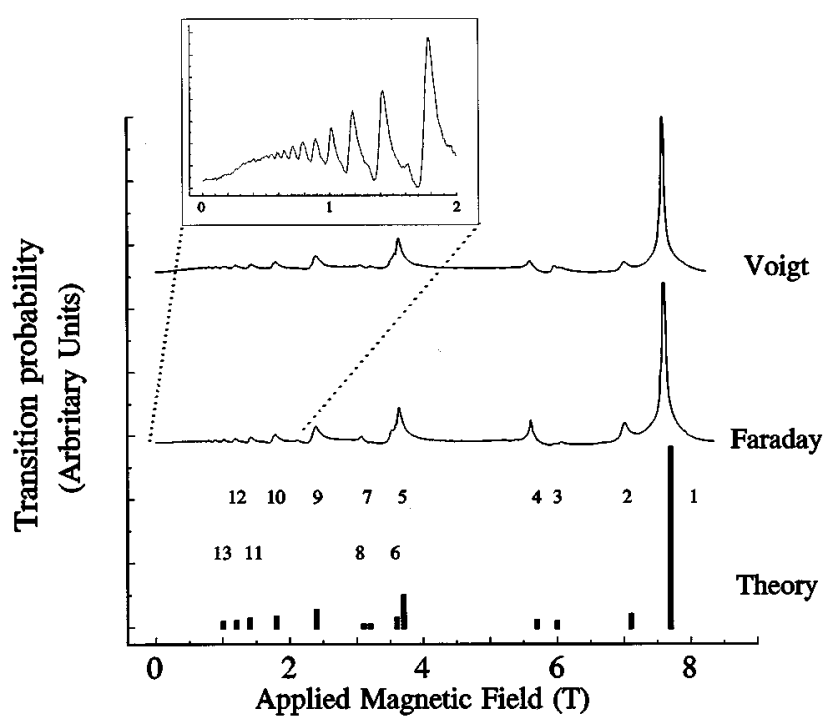

FIG. 5. A plot of experimental transition spectra for the Faraday and Voigt experimental configurations at an applied laser wavelength of $70 \mu \mathrm{m}\left(\equiv 142.86 \mathrm{~cm}^{-1}\right)$ and a theoretical spectrum at the same transition energy. The lines are numbered as follows: $1=(1,1,0), 2=(1,-1,0), 3=(1,0,1), 4=(1,1,2), 5=(2,1,0), 6=(2$, $-1,0), 7=(2,0,1), 8=(2,1,2), 9=(3,1,0), 10=(4,1,0), 11=(5,1,0)$, $12=(6,1,0), 13=(7,1,0)$. 


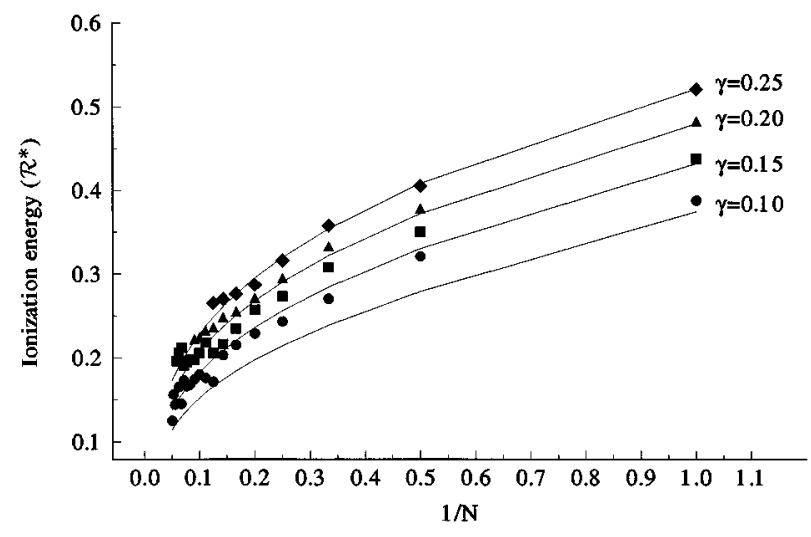

FIG. 6. Theoretical and experimental ionization energies against $1 / N$ for differing values of $\gamma$.

the same size as the large transition peak in the experimental spectra. It can be seen that the pattern of predicted transitions follows closely the peaks in the experimental spectra both in the value of the magnetic field at which the transitions takes place and in the comparative strength of the transitions. The similarities between the actual and predicted spectra provides further evidence of support for the validity of the model.

Figure 5 also shows that the method can be used as a tool for the identification of the peaks observed in experimental spectra. It is interesting to note that both transitions to the odd parity states [namely the $(1,0,1)$ and $(2,0,1)$ peaks] are seen to be more prominent in the Voigt geometry spectrum, as expected. Also, at lower magnetic fields, the spectra show the characteristic "oscillatory" behavior corresponding to the whole series of transitions to the $(N, 1,0)$ Landau-like states. An expansion of the scale of the spectra at these low fields, included as an insert in Fig. 5, shows transitions to at least the $N=10$ Landau-like state. This appears to be the first example in which such highly excited states have been reported for impurities in bulk GaAs.

\section{B. Highly excited states}

We can use the availability of experimental data involving highly excited impurity states to investigate the behavior of Landau-like states with $N$ greater than that considered previously in Figs. 1 to 4. A plot of the calculated and experimentally determined ionization energies against $1 / N$ for varying values of magnetic field $\gamma$ for states with $m=1$ and $\nu=0$ is given in Fig. 6. The experimental data have been obtained through the interpolation of data from the magnetic-field scans at fixed laser frequencies. For the largest fields considered, the theoretical curves agree with the experimental data for the states with lower values of $N$. However, for states with higher Landau level numbers $N$, the experimental values of the ionization energies are consistently higher than the theoretical predictions. Also, the agreement is generally poorer for the lower fields. Although the better agreement at higher fields was to be expected, the deterioration with increasing values of $N$ appears to be contradictory. As noted in Sec. IV, the model should become more accurate for increasing values of $N$ where the electron is less tightly bound to the central ion by the Coulomb interaction. As the agreement with the experimental data for low $N$ is good, we feel that this discrepancy is not due to the breakdown of the validity of the theoretical model but rather that the origin of the inaccuracies in the data for states of high $N$ is due to problems with the analysis of the experimental results.

Any transitions to the Landau-like states are relatively broad because of lifetime broadening of the excited states originating from the autoionizing behavior of these states. ${ }^{22}$ This leads to non-negligible errors in the determination of the experimental transition fields. Because the ionization energies of states with large $N$ are rather small, an inaccuracy in the value of the field at which the transition occurs leads to a relatively large error in measuring the ionization energy. This conclusion is supported by the large spread in the experimental data for high values of $N$ which is clearly visible in Fig. 6.

One reason for a consistent shift of the experimental data to energies higher than that predicted from the model may be due to the large transition energies involving the highly excited states. It is possible that the expressions of Peeters and Devreese, ${ }^{18}$ which are valid at low fields, underestimate the effects of the electron-phonon interaction for states of high $N$. The resonant electron-phonon interaction will result in a lowering of transition energies below the longitudinal-optical phonon energy, which in turn would give larger values for the measured ionization energies. A second origin for the discrepancy between theory and experiment may be due to the asymmetric line shape observed in transitions towards the Landau-like states of high $N$ (see insert in Fig. 5). The Landau-like impurity states exist only in an external magnetic field and experience strong interference with the continuum of free-electron Landau states. This is often referred to as Fano resonance. ${ }^{23}$ This interference is the origin of the autoionizing character of these states and leads to the characteristic asymmetric line shape of the transitions with an absorption minimum on the low-field side of the peak. The maximum in the optical absorption, or the maximum in the optically induced conductivity observed in these experiments, does not occur at the resonant field for the discrete donor state but rather at a somewhat higher field. In order to extract the correct field values from the experimental data, the actual line shapes have to be analyzed in terms of the coupling between the discrete and continuum states and the various optical transition probabilities.

Because of frequent (partial) overlap of other neighboring transitions and the lack of a priori knowledge on interaction strengths, a detailed analysis of Fano resonance is beyond present capabilities. It is clear, however, that the transition fields used for the data in Figs. 1-4 and Fig. 6, which have been obtained from the measured maxima in the photoconductivity, are higher than the correct values. This will result in a systematic overestimation of the calculated ionization energies. A rough fitting of the line shapes of the high $N$ transitions to that of the $(2,1,0),(3,1,0),(4,1,0)$, and $(5,1,0)$ states at higher fields, where overlap between lines causes a lesser problem, indicates that the error in the obtained ionization energies can easily be of the order of $0.5-2 \mathrm{~cm}^{-1}$ $\left(0.01-0.04 \mathcal{R}^{*}\right)$. This compares well with the deviations between the experimental and numerical results shown in Fig. 6 , which are all of this magnitude. These systematic errors will be more pronounced for low-field values because the interaction between the discrete and continuum states will be expected to be stronger than in higher fields, closer to the 
TABLE II. Theoretical values of ionization energy of $(2, m, 0)$ states at magnetic field of $\gamma=2$ and 5000 .

\begin{tabular}{lcc}
\hline \hline$m$ & Ionization energy $(\gamma=2)$ & Ionization energy $(\gamma=5000)$ \\
\hline-1 & 0.85205 & 13.35505 \\
0 & 0.99850 & 14.65936 \\
1 & 0.968138 & 14.62408 \\
2 & $0.932877(0.94234)$ & 14.58907 \\
\hline \hline
\end{tabular}

adiabatic limit. This trend is observed in Fig. 6. We therefore conclude that the observed deviations between the theoretical and experimental ionization energies of highly excited impurity states can be largely attributed to the interference effects between bound donor states and the continuum of Landau states (i.e., the occurrence of Fano resonances in the spectra of the highly excited Landau-like states).

\section{DISCUSSION OF THE ORDERING OF LANDAU-LIKE STATES}

It is interesting to calculate the ionization energies of the impurity states associated with a given Landau level $N$ for different values of the quantum number $m$. Table II gives the results for some of the $N=2, \nu=0$ states at magnetic fields with $\gamma=2$ and 5000. The ordering obtained is different from that given by Simola and Virtamo, ${ }^{4}$ who found that the levels for which $m=-1,0,1$, and 2, respectively are in order of increasing ionization energies. In contrast, we find that the energies of the $m=1$ and 0 states are larger than that of the $m=2$ state and that the ordering is the same even at the very high magnetic field considered $(\gamma=5000)$. For comparison, the calculation by Friedrich ${ }^{10}$ of the ionization energy for $\gamma=2$ is given in brackets in Table II for the hydrogenic state $(2,2,0)$; note that any inaccuracies in modeling the $m=1,0$ states would have resulted in lower values of ionization energy rather than higher values.
Figure 7 shows three-dimensional plots for some of the wave-function probabilities $\Psi_{N m \nu}^{*} \Psi_{N m \nu}$ for the $(2, m, 0)$ states. It can be seen that the electron in the $m=0$ state is more localized around the central ion than in other states. Thus we would expect it to have the highest energy, as is indeed obtained in contradiction to Simola and Virtamo. ${ }^{4}$ It is interesting to note also that the order of levels obtained here for bulk GaAs is the same as that obtained by us using the totally different method ${ }^{24}$ developed for a quantum well (QW).

\section{CONCLUSION}

A theoretical description of a shallow donor impurity in a magnetic field which uses the adiabatic approximation and employs a simple variational procedure to describe the behavior of the donor electron has been outlined in this paper. Corresponding experimental data have also been obtained for GaAs doped with silicon. Though the model is not necessarily suitable for the description of the hydrogenlike behavior of the impurity, it has proved to be very successful in describing the Landau-like donor electron states. It predicts accurately the ionization energies of these states. Since the method generates a simple form of the impurity wave function, it has been seen to be a useful tool in identifying the many transitions in experimental spectra involving the Landau-like states of the donor impurities. However, our discussion highlights a few discrepancies in the ordering of some of the states compared to that obtained originally by Simola and Virtamo. ${ }^{4}$

Whereas the above details were set up specifically for a donor impurity in bulk GaAs, many of the results and features have a more general applicability. For example, they can also be applied to hydrogen atoms in stars as discussed, for example, in the review by Friedrich and Wintgen. ${ }^{13}$ Another area of much current interest is that of donor impurities in multi-quantum-well (MQW) systems such as that formed

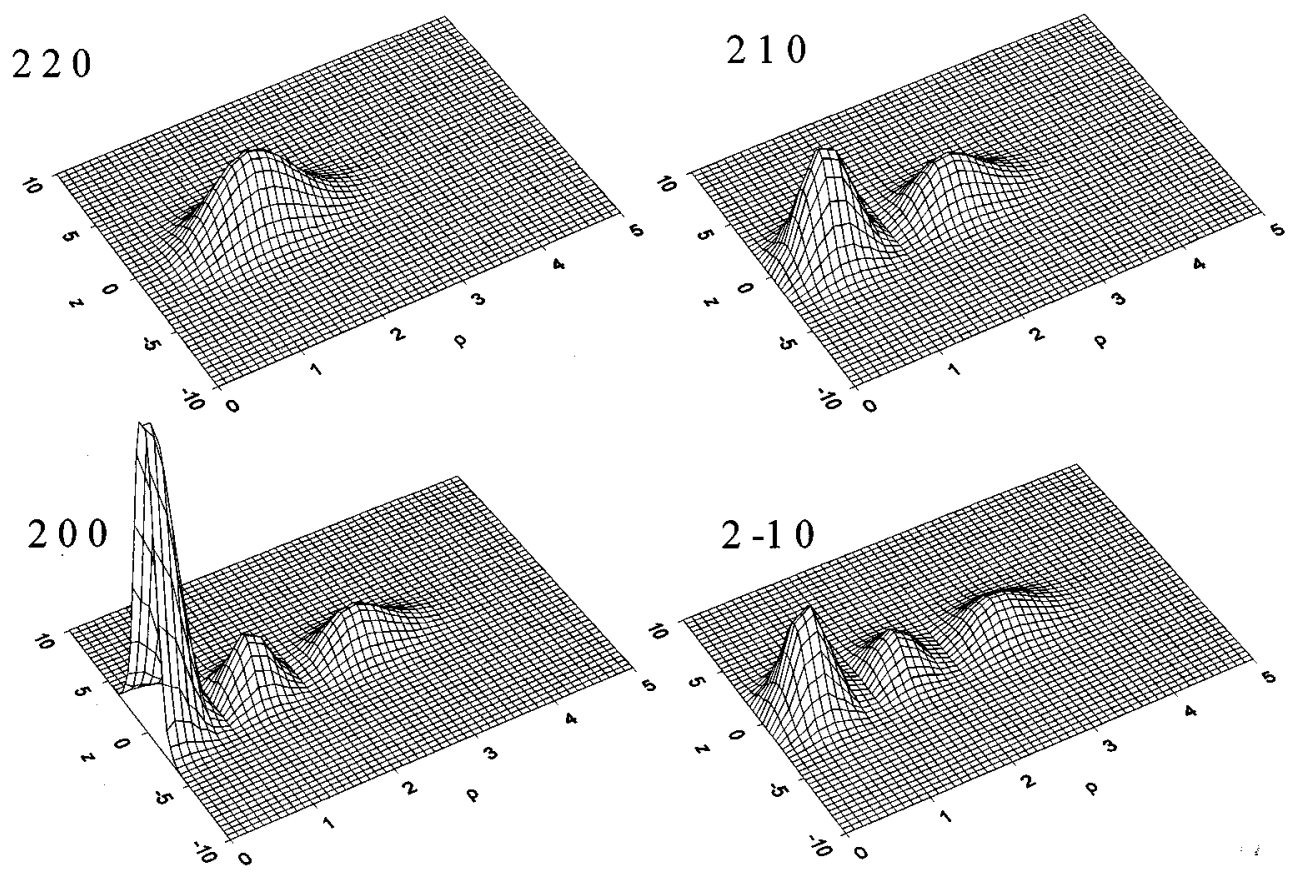

FIG. 7. Plots of the wavefunction probabilities for the $(2,2,0), \quad(2,1,0), \quad(2,0,0), \quad$ and $(2,-1,0)$ impurity states for a magnetic field of $\gamma=2$. 
by layers of $\mathrm{GaAs} / \mathrm{Ga}_{x} \mathrm{Al}_{1-x} \mathrm{As}$ grown by, for example, MBE methods. The most recent work here is that of Chen et $a .^{25}$ for simple donors in QW's and by Bruno-Alfonso et $a l^{26}$ for conduction electrons in superlattices in the presence of magnetic fields. In both cases, magnetic fields were present. The $D^{-}$donor in GaAs QW's has also been studied very recently by optically detected far-infrared resonance experiments $^{27}$ and theoretically ${ }^{28}$ by including the electronphonon interaction. However, only hydrogenlike states ap- pear to have been considered in these analyses.

Work has already started to extend the method developed here for Landau-like levels in bulk semiconductors to MQW systems. However, two of the authors (J.L.D. and C.A.B.) have already been involved in other theoretical work on donors in MQW systems, namely, those involving a nonvariational method ${ }^{24}$ and also an analytical method. ${ }^{29}$ The latter was an extension of earlier work on bulk systems described previously. $^{8}$
${ }^{1}$ H. P. Wagner and W. Prettl, Solid State Commun. 66, 367 (1988).

${ }^{2}$ A. van Klarenbosch, T. O. Klaassen, W. Th. Wenckebach, and C. T. Foxon, J. Appl. Phys. 67, 6323 (1990).

${ }^{3}$ S. J. Hawksworth, R. T. Grimes, E. P. Pearl, M. B. Stanaway, J. M. Chamberlain, J. L. Dunn, C. A. Bates, S. P. Najda, C. J. G. M. Langerak, J. Singleton, and C. R. Stanley, Semicond. Sci. Technol. 7, 1499 (1992).

${ }^{4}$ J. Simola and J. Virtamo, J. Phys. B 11, 3309 (1978).

${ }^{5}$ H. Friedrich and M. C. Chu, Phys. Rev. A 28, 1423 (1983).

${ }^{6}$ M. C. Chu and H. Friedrich, Phys. Rev. A 29, 675 (1984).

${ }^{7}$ D. Wintgen and H. Friedrich, J. Phys. B 19, 991 (1986).

${ }^{8}$ A. G. Zhilich and B. S. Monozon, Sov. Phys. Solid State 8, 2846 (1967)

${ }^{9}$ V. G. Golubev, V. I. Ivanov-Omskii, A. V. Osutin, R. P. Seishan, Al. L. Éfros, and T. V. Yaseva, Sov. Phys. Semicond. 22, 896 (1988).

${ }^{10}$ H. Friedrich, Phys. Rev. A 26, 1827 (1982).

${ }^{11}$ S. K. Bhattacharya and Shih-I. Chu, J. Phys. B 16, L471 (1983); 18, L275 (1985).

${ }^{12}$ C. H. Greene, Phys. Rev. 28, 2209 (1983).

${ }^{13}$ H. Friedrich and D. Wintgen, Phys. Rep. 183, 37 (1989).

${ }^{14}$ R. F. Wallis and H. J. Bowlden, J. Phys. Chem. Solids 7, 78 (1958).

${ }^{15}$ A. J. van der Sluijs, K. K. Geerinck, T. O. Klaassen, and W. Th. Wenckebach, Proceedings of the 18th International Conference on Infrared and Millimetre Waves, Colchester, UK, 1993, edited by J. R. Birch and T. J. Parker [SPIE 2104, 513 (1993)].

${ }^{16}$ P. W. Barmby, J. L. Dunn, C. A. Bates, E. P. Pearl, C. T. Foxon, A. J. van der Sluijs, K. K. Geerinck, T. O. Klaassen, A. van Klarenbosch, and C. J. G. M. Langerak, J. Phys. Condens. Matter 6, 7867 (1994).

${ }^{17}$ Q. H. F. Vrehen, J. Phys. Chem. Solids 29, 129 (1968).

${ }^{18}$ F. M. Peeters and J. T. Devreese, Phys. Rev. B 31, 3689 (1985).

${ }^{19}$ A. van Klarenbosch, Ph.D. thesis, University of Leiden (The Netherlands), 1990.

${ }^{20}$ P. C. Makado and N. C. McGill, J. Phys. C 19, 873 (1986).

${ }^{21}$ J. L. Dunn and E. P. Pearl, J. Phys. Condens. Matter 3, 8605 (1991).

${ }^{22}$ A. van Klarenbosch, J. Appl. Phys. 67, 6323 (1990).

${ }^{23}$ U. Fano, Phys. Rev. 124, 1866 (1968).

${ }^{24}$ T. Kuhn, G. Mahler, J. L. Dunn, and C. A. Bates, J. Phys. Condens. Matter 6, 757 (1994).

${ }^{25}$ R. Chen, J. P. Cheng, D. L. Lin, B. D. McCombe, and T. F. George, J. Phys. Condens. Matter 7, 3577.

${ }^{26}$ A. Bruno-Alfonso, L. Diago-Cisneros, and M. de Diosa-Leyva, J. Appl. Phys. 77, 2837 (1995).

${ }^{27}$ J. Kono, S. T. Lee, M. S. Salib, G. S. Herold, A. Petrou, and B. D. McCombe, Phys. Rev. B 52, 8654 (1995).

${ }^{28}$ J. M. Shi, F. M. Peters, and J. T. Devresse, Phys. Rev. B 51, 7714 (1995).

${ }^{29}$ B. S. Monozon, C. A. Bates, J. L. Dunn, and M. J. Pye, Phys. Rev. B 53, 12938 (1996). 